\title{
A Proposal for Sleep Scoring Analysis Designed by Computer Assisted using Physiological Signals
}

\author{
Hemu Farooq, Anuj Jain, V.K. Sharma
}

\begin{abstract}
Sleep is utterly regarded as compulsory component for a person's prosperity and is an exceedingly important element for wellbeing of a healthy person. It is a condition in which an individual is physically and mentally at rest. The conception of sleep is considered extremely peculiar and is a topic of discussion and researchers all over the world has been attracted by this concept. Sleep analysis and its stages is analyzed to be useful in sleep research and sleep medicine area. By properly analyzing the sleep scoring system and its different stages has proven helpful for diagnosing sleep disorders. As it's seen,sleep stage classification by manual process is a hectic procedure as it takes sufficient time for sleep experts to perform data analysis. Besides, mistakes and irregularities in between classification of same data can be recurrent. Therefore, theuse of automatic scoring system in order to support reliable classification is highly in greater use. The scheduled work provides an insight to use the automatic scheme which is based on real time EMG signals and Artificial neural network. EMG is an electro neurological diagnostic tool which evaluates and records the electrical activity generated by muscle cells. The sleep scoring analysis can be applied by recording Electroencephalogram (EEG), Electromyogram (EMG), and Electrooculogram (EOG) based on epoch and this method is termed as PSG test or polysomnography test. The epoch measured has length segments for a period of 30 seconds. The standard database of EMG records was gathered from various hospitals in sleep laboratory which gives the different stages of sleep. These are Waking, Non-REM1 (stage-1), NonREM2 (stage-2), Non-REM3 (stage-3), REM. The collection of datawas done for the period of 30 second known as epoch, for seven hours. The dataset obtained from the biological signal was managedso that necessary data is to be extracted from degenerated signal utilized for the purpose of study. As a matter of fact, it is known electrical signals are distributed throughout the body and is needed to be removed. These unwanted signals are termed as artifacts and they are removed with the help of filters. In this proposed work, the signal is filtered by making use of low-pass filter called Butterworth. The withdrawn characteristics were instructed and categorized by utilizing Artificial Neural Network (ANN). ANN, on the other hand is highly complicated network and utilizing same in the field of biomedical when contracted with electrical signals, acquired from human body is itself a novel. The precision obtainedby the help of the procedure was discovered to be satisfactory and hencethe processis very useful in clinics of sleep, especiallyhelpful for neuro-scientists for discovering the disturbance in sleep.
\end{abstract}

Key Words: Sleep Scoring Analysis, Stages of sleep, EEG, EMG, EOG, Artificial Neural Network (ANN)

Manuscript received on May 10, 2021

Revised Manuscript received on June 12, 2021.

Manuscript published on June 30, 2021.

* Correspondence Author

Hemu Farooq*, Department of Electronics and Communication Engineering, Bhagwant University, Ajmer (Rajasthan), India.

Anuj Jain, Department of Electronics and Communication Engineering, Bhagwant University, Ajmer (Rajasthan), India.

V.K. Sharma, Department of Electronics and Communication Engineering, Bhagwant University, Ajmer (Rajasthan), India.

(c) The Authors. Published by Blue Eyes Intelligence Engineering and Sciences Publication (BEIESP). This is an open access article under the CC BY-NC-ND license (http://creativecommons.org/licenses/by-nc-nd/4.0/)

\section{INTRODUCTION}

\section{A. SLEEP Scoring Analysis: -}

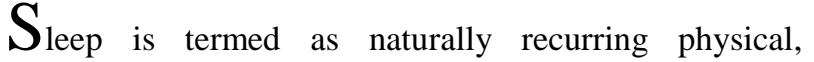
psychological and emotional situation of a human being which is prominent by altering awareness. Sleep includes interference of all volitional muscles and hence there is a minimum interaction with the surrounding happenings [1]. Across animal evolution, sleep is known to be favorably conserved behavior. Sleep is thestate in which an individual experiencesthe condition from conscious to sub conscious phase. In many physiological processes, sleep is regarded as necessary phase which includes experience processing as well as memory consolidation. Persons affecting from sleep ailments like sleep narcolepsy, insomnia and sleep nocturnal breathing etc. do not get sufficient sleep and thus need to be attended well. Thus, importance of sleep is stressed by the manifestation of processesfor those who experience sleep disorders [2].

In front of sleep expert, mechanism of scoring procedure is done by inspecting and examining the PSG test (polysomnography). Sleep scoring mechanism is considered as animportantcharacteristic in the field of research and medicinal study.PSG (Polysomnography), on the other hand is an experiment which involves record transference of multiple physiological signals like Electroencephalogram (EEG), Electromyogram (EMG) and Electrooculogram (EOG). The biologicalwave which was recorded areacquired carefully for the small division of time called as epoch, and when inspected gives the various sleep stages [3].

Usually,classification of sleep scoring analysis distinguishes sleep into various types of stages such as Wakefulness, REM (Rapid Eye Movement), NREM (NonRapid Eye movement). Wakefulness is known as the state where a person is preparing for sleep. It is that particular state, where a person is in relaxed and soothe mode, and also EMG of a subjectfeasibly is upraised or in average mode. Non-Rapid Eye movement are additionallysegmented into four stages i.e., stage First: which is the state after small time is consumed in wakefulness stage and EMG is declined, stage Two: is that stage of sleep in which a person asleep and EMG value further decreases, stage Third: in which EMG gets further declined due to muscle relaxation, stage Fourth: which records the moderate EMG and a person is termed asleep. Rapid Eye movement is the stage in which EMG indicates the muscle tones termed as twitches and is esteemed by rapid or random movements of eye [4].

Blue Eyes Intelligence Engineering and Sciences Publication

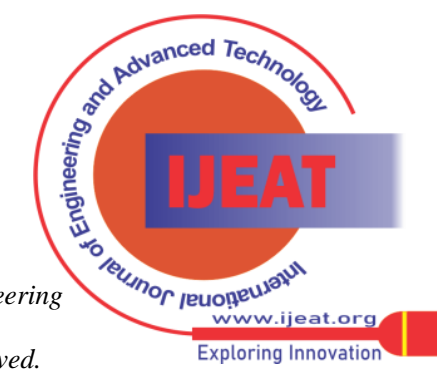


Sleep is an important aspect for persons good health and is appraised as important element for both mental and physical ability of an individual. Sleep is virtual detachment of a human being from his surroundings. Sleep is the condition when both mental and physicalconditions of an individual are in a state of rest. The conception of sleep is intended to be extremely curious all over the globe which has attracted the scientists to work on it [5]. Insufficiency of sleep ordisorders prompts numerousailments of heart, kidney, etc. and enlarges the possibility of disorders like hyperglycemia, hypertension as well as brain strokes. These diseases are called as passive diseases. Thus, disorders caused by inappropriate sleep need to be handled [6].

Sleep scoring system is of great significance in the study of sleep medicine and has gained name in the research of sleep. Automaticscoring system has been undertaken by the visual investigation of polysomnograms (PSG). PSG test is the conduction of various biological signals like EOG (Electrooccularogam), EMG (Electromyogram), EEG (Electroencephalograph) which when examined only for a small amount of time known as epoch and analyzed gives us different sleep stages $[7,8]$.

\section{B. PhysiologicalSignals}

Electroencephalogram (EEG): The electrical activity of the brain can be evaluated with the help of an electroencephalogram (EEG) test. The stimulated exercise of the brain is the connection between the cells of brain through impulses in electric form. The waves of brain pattern are traced and listed by using EEG. The electrodes used for analyzing the electrical impulses are fixed to the scalp with the help of wires. These electrodes are small flat discs and are used to record impulses in the brain in order to send signal to computer. The impulses in EEG recordings will have wavy lines along with valleys and peaks. With the help of these waveforms the doctors are able to assess if the patterns are normal or abnormal. Any abnormalities found will be a sign of seizures or brain disorders.

Electromyogram (EMG): It is a diagnostic procedure for evaluating and controlling the health conditions of the muscles as well as the nerve cells (motor neurons). The translation of the signals into numbers or graphs is done by the EMG thus helping the doctors in the process of diagnosing. If person visits the doctors with the symptoms of nerve or muscle disorder which include the tingling, numbness, or unexplained weakness in the limbs the doctors will suggest an EMG since its results will assist the doctors in diagnosing the disorders of the muscles, nerves or the disorders which is affecting the connection amidst the muscles and nerves.

Electrooculogram (EOG): This method is used to measure thepotential of corneo-retinal which is present amid the back and front of the human eye. The resultant signal is called as the electrooculogram. EOG is usually applied for diagnosing the ophthalmological issues and for recording the movements of the eyes. For measuring the movement of the eye, the electrodes are allocatedabove or belowandright or left of the eye respectively. When the eye proceeds from the center to any one of the electrodes, it shows the positive side of the retina while the other electrode will show the negative side of the retina hence developing a potential difference between the electrodes. The potential which is recorded will be the measure of the position of the eye.

\section{ANN (Artificial Neural Network)}

Retrieval Number: 100.1/ijeat.E26090610521

DOI:10.35940/ijeat.E2609.0610521

Journal Website: www.ijeat.org
Artificial Neural Network (ANN) is appreciablyinvolved in the approach of computing. It can be used for a spectacular form of processes like image recognition,recognition of pattern, and in analyze, classification of patterns. ANN has been widely famous in the world of computing. Inspite of measuring huge amount of data, ANN is exceedingly effective classifier in terms of ease designing, time execution and good accuracy [9].

The scheming and modelingwere achieved by the concept of biological neural system which is quite impressive. Biological neural system possess brain which is part of central nervous system, consisting of cells called as neurons having principal function of processing, assembling and distribution of signals. Thus, an artificial neural network is information processing unit which works in similar way as biological neural network. Mathematically, it can be termed as functioning executed by an activation factor while receiving input $\mathrm{x}$ and weight $\mathrm{w}$. It can be written as:

$$
\text { net }=\sum_{i=0}^{n} w_{i} x_{i}
$$

Where $x_{i}$ shows the input to neuron and $w_{i}$ shows weightwhich is relatable to input respectively. An activation function $f_{\text {act }}$ is implemented to net giving as a result the output of neuron $\mathrm{O}$ and amplitude of neuron in output is limited by this function [10].

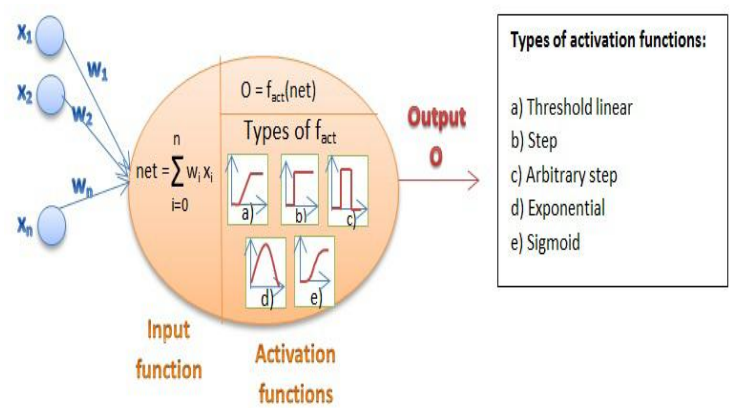

Fig. 1 An Artificial Neuron

The fundamentalattribution of artificial neural system when accomplished for measuringsource are its potentiality to modify as well as essential quality of multi-processor memory and non-algorithmic function. It consists of three operational layers vizinput layer, output layer and hidden layers respectively. Also, it is been observed that there can be chance of some supplementary middle layers which fulfils the gathering of multipleduty. The supplementary middle layers are made up of cells whose main purpose is to carryout is addition which is a consequence of previous layer emanated by output [11]. The hidden layer resolution is the critical work in terms of outlining and figuring the neural networks. By employing hit and trial method, thecomputation of middle layers and its activation factor are done reasonably by ANN. This period of method is known as training phase and as per calculation, trained function of neural network is acquired.

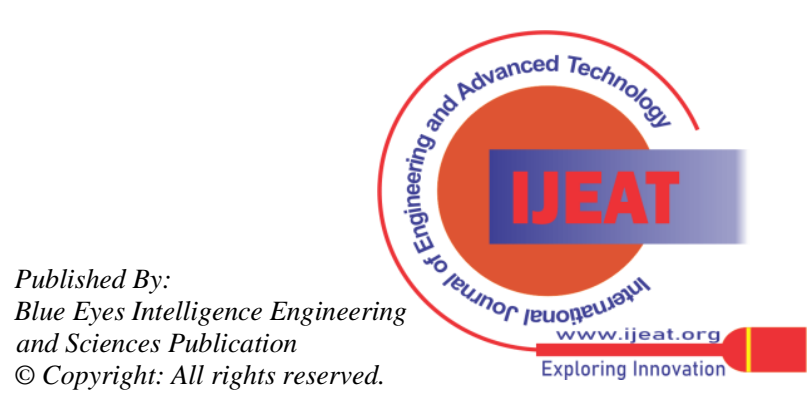




\section{MATERIAL METHODS}

The real time implementation was used for the development of the research.

\section{a. Equipment's and devices}

There were interfacing between the polysomnography and personal computers.

- Data collected for the studying of sleep stages were recorded through device called polysomnography device. The data was recorded for the period of seven hour for each subject. These data which were variant was collectively first grouped by sleep experts and polysomnography software device.

- A designed biomedical device designed for computation of physiological signals especially during phases of sleep.

\section{b. Materials}

Electroencephalogram (EEG)

Electromyogram (EMG)

Electrooculogram (EOG)

- Sleep Electroencephalogram Recognition NN (SRNN): enables to detect a variety of essential characteristic features in EEG waveform.

- $\quad$ Sleep Stage Diagnosis NN (SSNN): enables to diagnose different stages of sleep.

- Contextual Diagnosis Neural Network (CDNN): for stimulating the correct decision rate by considering contextual.

- Relation of performance of characteristic waves.

The execution is established on ANN (Artificial Neural Network) classifier using MATLAB software.

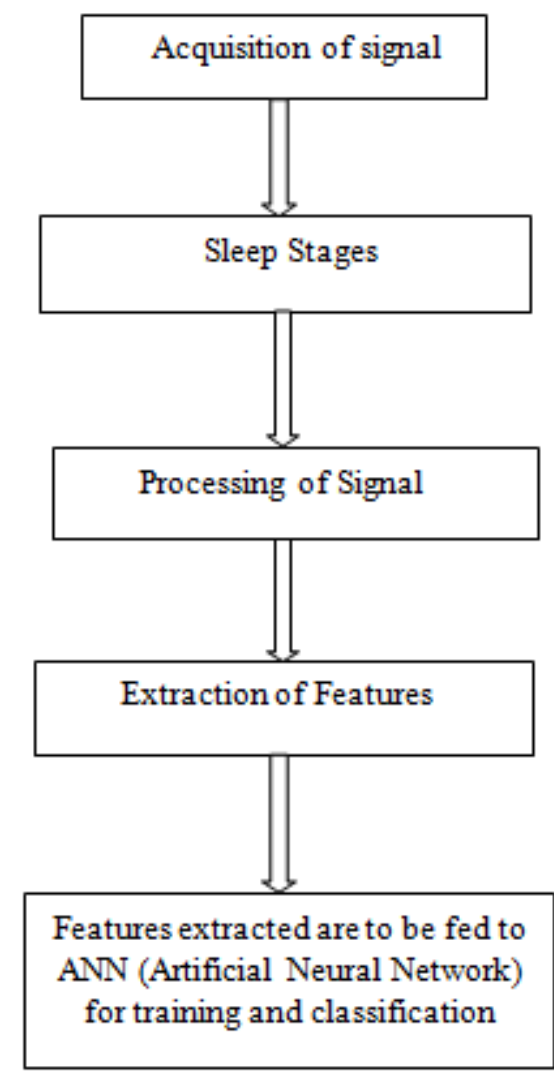
confirm the supremacy of the proposed study employing Artificial Neural Network (ANN).

\section{RESULTS}

For the better quality of life and good health, sleep plays a key part and preserves physical and mental health of a person throughout their living. There are so many peoples who are experiencing such sleep disorders which affect their daily life. These sleep disorders are sleep apnea, sleep insomnia and narcolepsy etc. The analysis of sleep stages are thus useful for recognition and care of such type of disorders need to be implemented.

In front of sleep specialists, different quality of dataset from biological signal was acquired from various sleep laboratories in different hospitals. The different physiological signals were collected in real time like EEG signal were collected in a standard way by using electrodes on the skin surface. While EMG signal were achieved by keeping two electrodes below the chin of a subject. Whereas EOG signal were collected by using electrodes positioned above or below and right or left of the eye respectively. Thus, the physiological signal records obtained after analyzing the process gives clear understanding of different sleep stages. These are followed as:

\section{a. SignalProcessing:}

The original data acquired from EMG signal has to be manipulated which is required for using the Artificial Neural Network. The signals are processed to remove corrupt signals, extraction of crucial information and to predict values. Physiological signals are frequentlyaltered by other waves which are not at all associated. Physical or technica affair are the reason for these signals. A human body produces its own electrical activities and it can be found using the following devices.

- Artifacts in EEG - spike occurrence in the stage of QRS complex.

- Artifacts in EOG caused by blinking and eye movements.

- EMG has high-frequency artifacts.

The general possible process is to abolish these artifacts by using filters such as low-pass filters, high-pass filters, and band-pass filters, depending on the electrical disturbance present in signal. The signal re-processingis accomplished to improve the EMG signal by the process of normalization. EMG signal is segmented into 30 seconds of part called as epoch,and with each epoch correlatesthe different stages of sleep. In this, the signals are filtered using butterworth Low pass filter (LPF).

\section{b. Artificial Neural Network.}

Artificial Neural Networkare figured as motivated from neural systems in human beings. It is seen, when compared to the human structural system, ANN has more easy formation having some attributesconstitutes learning and reasoning. On the other hand, other attributes are however perceivedwith the approaches of planning instead of neuropsychological ones [13]. 
The basic attributes of neural networks presently put into application are existence of non-algorithm, paralleldistributed memory and adaptive [12]. Artificial Neural Networks are presently used in investigation, classing, recognition of patterns and monitoring of signals and systems which are functional [14]. Neural networks have been fortunate inpreparing of physiological signals to a greatscope, incorporating during cognitive load, rest, sleep of EEG signal for better examination and explanation [15, 16]. Usually, an Artificial neural network comprises of input layer, output layer each one in number and hidden layer which may be one or more than one. The layers consist of cells which performs the summation of outputs given by previous layers through the neurons multiplied with weighting vector. Thus, output is produced with a non-linear transfer function known as activation function for every node or cell.There are other researches going on this topic which has given satisfactory results, ANN in this study is used as classifier and it was realized using MATLAB.

The variety ofeach epoch in sleep usedvary in accordance withmixture of stages of sleep that were used for classification. Stage of sleep were always utilized for training phase and testing phase. The dataset was segmented into the training set which consists of $60 \%$ of the data,20\% of the data was used as the validating set, and the other $20 \%$ was used to testing phase of network. Three different classifications of the data were approached. The first classification differs only between the W stage, N1 stage, and REM stage, while the second alternative classifies $\mathrm{W}$, $\mathrm{N} 1, \mathrm{~N} 2$, and REM sleep stage, and the third option scores all the sleep stages. The importantsegment of the neural network is the input matrix and the target matrix.

The input matrix used to train the ANN is the training set and toadjust the neural network is the target set according to differences between the output and the target of the neural network. The input data were segmented into training, validating and testing set using the function dividerand, in ratios $60 \%$ training set and remaining $40 \%$ the testing set. The target matrix consists of zeroes and ones, as one is only in the column representing the correct stage marked by a sleep expert.

To generate the ANN itself, the function nftoolopened the ANN generator in Matlab.The input variables, target and input, were chosen along with the allocation of the training and testing set. The training function of the network is the algorithm of Levenberg-Marquardt optimization of weights and bias update. Even though the algorithm needs more memory than other algorithms, it was selected due to its speed. To create the ANN structure (Fig. 6.1) the number of hidden neurons had to be chosen. The output was is the variable net with all the networks parameters in it. The output arguments, net and tr, represent the adjusted network (net), the

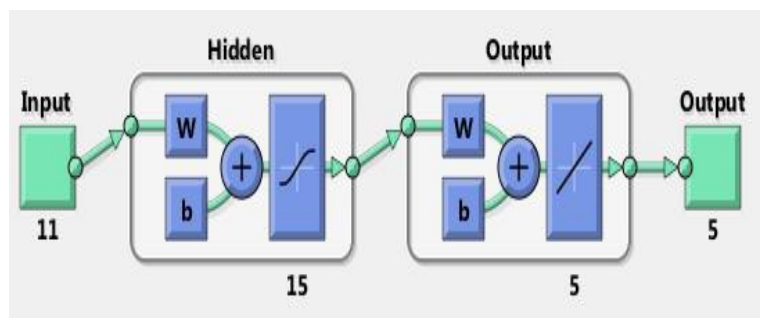

Figure 6: The structure of the ANN.
Number of the learning epochs (epoch), and the momentary performance. The networks' performance was set using the minimum square error method. To statistically evaluate the accuracy and performance of the ANN the confusion matrix (Tab 6) provides general overview of results compared to targets in percent's. The rows of this matrix represent the actual class and the columns usually represents the predicted class.

\section{c. Results of Proposed Model}

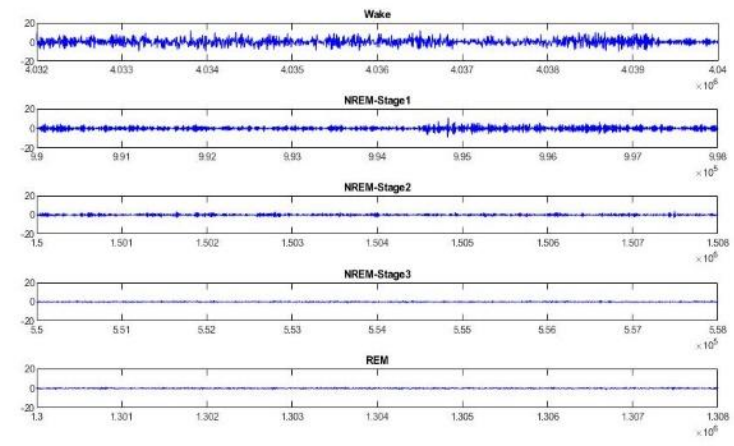

Figure 7: EMG signal acquisition

The above figure shows the different stages of sleeps with the activity of the brain.

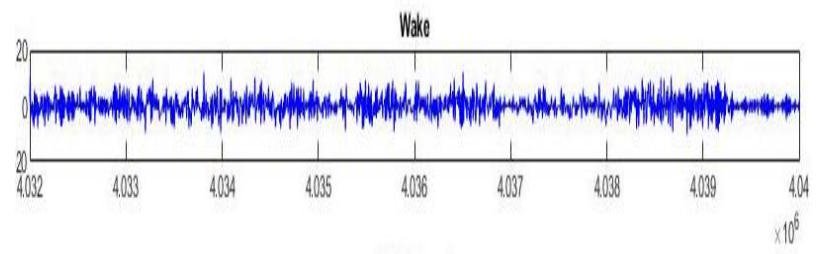

Figure 8: Activity during wakefulness

Wakefulness or (Stage 0): It is explained as the situation where a person closes his/her eyes and body in calm position or it is termed as state of living before starting of sleep. In this momentary stage, following things happen; (a) The EMG normally occur with beta wave of bandwidth (16$25 \mathrm{~Hz})$, amplitude (10-34 $\mu \mathrm{V})$ and higher amplitude alpha wave $(8-12 \mathrm{~Hz})$. When a person falls asleep, theta wave with amplitude (4-8 Hz) controllably occurs [17]. (b) The range EMG may be higher or moderate determined by amount of muscle tension [15]. REMs determined by usual scanning may be hard or short [16].

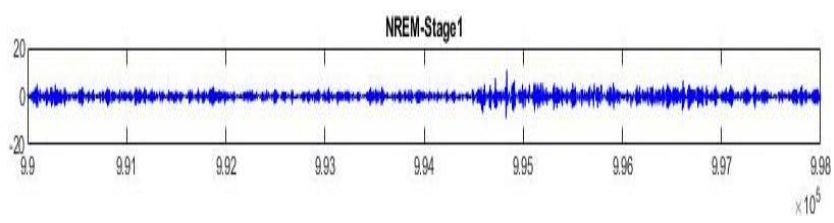

Figure 9: Activity during drowsy

Stage First: It is the stage of short period. The EEG in this stage occurs with repressed alpha wave which happen to be less than $50 \%$ of record, composed of harmonics with lower amplitude fluctuating between $3-7 \mathrm{~Hz}$ [16].

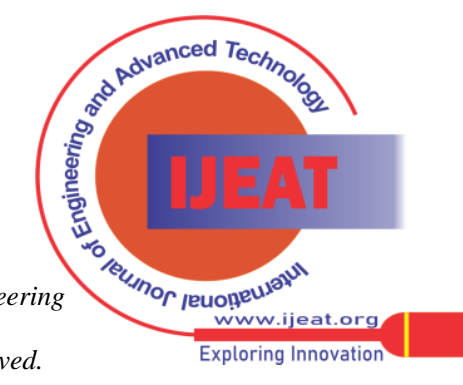


On the other hand, EMG value decreases than that in stage 0 [14] and REM stars gradually decreasing.

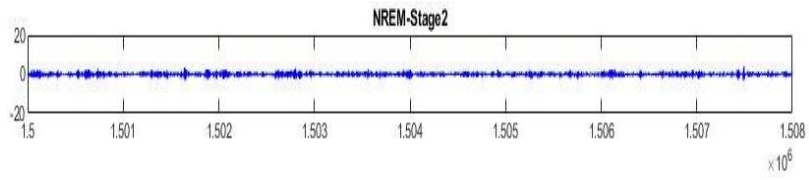

Figure 10: Activity during light sleep

Stage Second: In this stage, EMG occurs decreased muscle tones [15] and REM disappears. EEG in this stage consists of flashed mixed frequency ranging between 2-16 $\mathrm{Hz}$ with low voltage in background. The waveform in this stage has sleep spindles and high voltage K-complexes called biphasic wave [17].

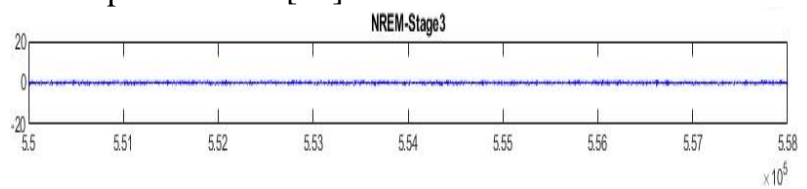

Figure 11: Activity during Deep sleep

Stage Three: In this stage, the EEG waveforms shows delta waves ranging between $0.5-4 \mathrm{~Hz}$ which are slow waves and high amplitude greater than $75 \mu \mathrm{V}$. The EMG in this stage has further decreased muscle tones. REM, on the other hand becomes in frequent except frequently artifacts which are due to other electrical disturbances in waveform.

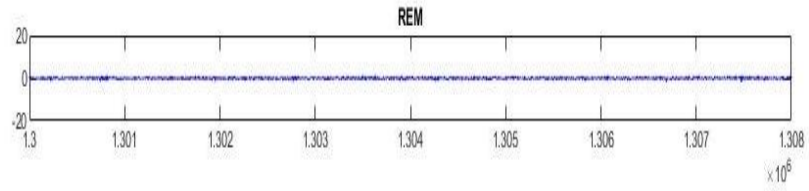

Figure 12: Activity during REM

REM stage: In this stage, EEG waveform regresses a low voltage spectral content which relates to that of wakefulness and stage first. The EMG in this stage consists of muscletones which are seldomly short tones called twitches. These twitches constitute transient activities arisen from blood pressure and high frequency [16]. The EOG on other hand shows dominant movements of eye [17].

\section{DISCUSSION}

An effectual proposal has been achieved by consideration of correlation of different stages of sleep and biological signal using highly classified and developed classifier known as Artificial Neural Network. The classifier via mathematical tool helps in determining the various stages of sleep. The main purpose of the proposed work is to ascertain different stages of sleep using biological signals viz EMG, EOG, EEG measured simultaneously and classified using ANN( Artificial Neural Network). The aim of the proposed work is to establish different stages of sleep and to calculate the efficiency of estimated performance metrices. As the accuracy achieved by Tagluk et al. [12] on capability of sleep scoring was about $74.7 \%$, which was taken as adequate when compared to the results obtained by Hanaoka et al. [14].

Synchronizing the signals of EEG, EMG, EOG has proved an effective alternative approach to ascertain the various stages of sleep. The utilization of EMG and EOC excluding artifacts from concurrent EEG signals was prosperous and thus it may be helpful in processing of all EEG signal studies. Several methods being initiated, beside the utilization ofthecomputer assisted system always entertain some advantage aboveequivalentstandard techniques and technology involved with the estimation of sleep stages. Most of these terminologies may include physiological stress on the subject which is being observed and it also requires specialists with advance quantity of competence and usage of a premium polygraphs. The estimation of sleep stages by $\mathrm{R}-\mathrm{K}$ (Rechtschaffen and Kales) have found as $82.6 \%$ for non-Rapid Eye Movement stages, 38.3\% for Rapid Eye Movement stage, 70.5\% for Awake stage [11]. In this case, the estimation of Rapid Eye Movement stage seems to be quiet low. An effort [12] of qualitatively clarifying the relation between sleep stages and heart rate are in fact considered not authentic categorization of sleep stages. Procedures [13] regarding the relationship between sleep stages and body movements were believed to be impractical in estimating the stages of sleep. Such noninvasive methods [14] that uses static charge sensitive bed does not define all types of sleep stages. The methods determined from movements of body measured by infrared sensors and extensive classifier has been prosperous in classifying the sleep stages. The aim of work by Akin et al. [15] and Kurt et al. [16] was to calculate the assertive mode and to calculate drowsy level respectively. However, in proposed work the motive is to classify the stages of sleep using EEG, EMG, EOG simultaneously when measured.

\section{CONCLUSION}

The aim of the study apart from the fact of considering the results acquired by comparativeanalysis,also involves a scheme that includes EEG, EMG, EOG signals to be fed to a classifier with a high precision that is Artificial Neural Network. The classifier ANN assisted by computer in analysis of sleep scoring system is the purpose of the study. In presence of sleep analyst, data base was acquired from different hospitals. Thus, physiological signals collected were first processed so that signals can be useful for the process and it was achieved by the normalization process. The essentialcharacteristic extracted were classified using Artificial Neural Network and the accuracy estimated by performance metrics is $83.33 \%$.

The motive of the work besides not only to consider at the results acquired by comparative analysis, but to provide a scheme also which involves EEG, EMG, EOG signals. In present work, the primary objective is the estimation ofstages of sleep using EEG, EMG, EOG when simultaneously measured. The process can be useful for scientists in inspecting the sleep and wake correlation and in diagnosing disorders of sleep.

\section{REFRENCES}

1. HJ. Park, JS. Oh, DU. Jeong, KS. Park "Automated Sleep Stage Scoring Using Hybrid Rule- and Case-Based Reasoning.", Computers and Biomedical Research , 33, pp. 330-349, 2000.

2. David T. Krausman, Bel Air, Richard P. Allen, "Sleep Scoring apparatus and method. Google Patents," 12 April 2005.

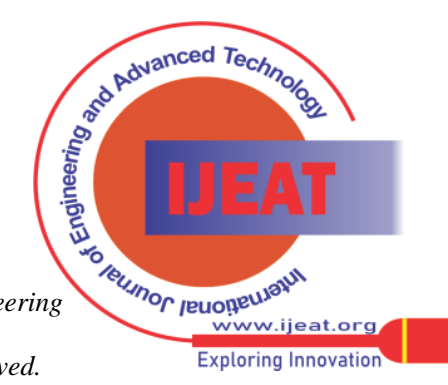


3. Penzel, T., Conradt, R., Computer based sleep recording and analysis. Sleep Med. Rev. 4:131-138, 2000.

4. Tagluk, M. E., Sezgin, N., and Akin, M., "Estimation of Sleep Stages by an Artificial Neural Network employing EEG, EMG, EOG", Journal of Medical System, 2010, 34(4), pp.717-725

5. ZT. Yeh, RP. Chiang, SC. Kang, CH. Chiang "Development of the Insomnia Screening Scale based on ICSD-II.", Int J Psychiatry Clin Pract , 16(4), pp. 259-267, Oct. 2012.

6. David T. Krausman, Bel Air, Richard P. Allen, "Sleep Scoring apparatus and method. Google Patents," 12 April 2005.

7. Vni Rao, M.D., Alyssa Bergey, B.A., Hugh Hill, M.D., David Efron, M.D., and Una McCann, M.D. Clinical Research Reports, Sleep Disturbance After Mild Traumatic Brain Injury: Indicator of Injury? he Journal of Neuropsychiatry and Clinical Neurosciences. 23: 2; 201-205, 2011.

8. Penzel, T., Conradt, R., Computer based sleep recording and analysis. Sleep Med. Rev. 4:131-138, 2000.

9. Tagluk, M. E., Sezgin, N., and Akin, M., "Estimation of Sleep Stages by an Artificial Neural Network employing EEG, EMG, EOG", Journal of Medical System, 2010, 34(4), pp.717-725.

10. MousmitaSarma, Kandarpa Kumar Sarma, "Fundamentals consideration of ANN", Phoneme-Based speech segmentation using hybrid soft computing frame work, April 2014; 47-75.

11. Kulkarni, P., Ade R., "Incremental learning from unbalanced data with concept drift and missing features: a review", International journal of data Min knowledge management process, 2014; 15-29.

12. Shimada, T., Tamura, K., Fukami, T., \&Saito, Y. (2010, July). The effect of using Elman-type feedback SOM for sleep stage diagnosis. In Complex Medical Engineering (CME), 2010 IEEE/ICME International Conference on (pp. 165-170). IEEE

13. Tagluk, M. E., Sezgin, N., \& Akin, M. (2010). Estimation of sleep stages by an artificial neural network employing EEG, EMG and EOG. Journal of medical systems, 34(4), 717-725.

14. Watanabe, T., and Watanabe, K., Noncontact method for sleep stage estimation. IEEE Trans. Biomed. Eng. 51 (10)1735-1748, 2004 doi:10.1109/TBME.2004.828037

15. Hanaoka, M., Kobayashi, M., and Yamazaki, H., Automated sleep stage scoring by decision tree learning, 23rd Annual EMBS International Conference, 1751-1754, 2001

16. Akin, M., Kurt, M. B., Sezgin, N., and Bayram, M., Estimating vigilance level by using EEG and EMG signals. Neural Comput. Appl. 17 (3)227-236, 2008. doi:10.1007/s00521-007-0117-7.

17. Kurt, M. B., Sezgin, N., Akin, M., Kirbas, G., and Bayram, M., The ANN- based computing of drowsy level. Expert Syst. Appl. 36 (2)2534-2542, 2009. doi:10.1016/j.eswa.2008.01.085.

\section{AUTHORS PROFILE}

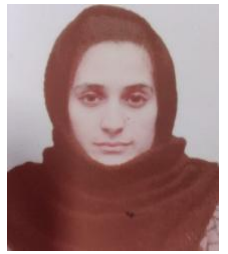

Hemu Farooq, is a $\mathrm{PhD}$ student at Bhagwant University, Ajmer Rajasthan pursuing $\mathrm{PhD}$ degree in electronics and communication engineering. She hold Masters of Technology from PTU and Bachelor's Degree from Kashmir University. She has published many papers. Her interests include biomedicine, artificial intelligence, Block chain, etc.

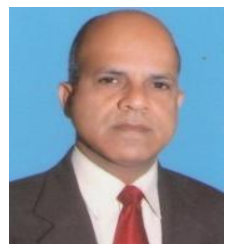

Prof. (Dr.) Virendra Kumar Sharma, was born in 1961. He received his B.E degree in Electrical Engineering from KREC (NIT), Surathkal, India in 1984 and received his M.Tech degree in Power Electronics from IIT Delhi, India in 1993. He received his Ph.D degree in the field from IIT Delhi, India in 2000 and he has done one year stint as PostDoctoral Fellow in Active Filters from ETS, Montreal Canada in 2001. Presently, he is a Professor in EEE and ViceChancellor of Bhagwant University, Ajmer, India since 2015. He is having total 35 years of teaching, research and academic administration experience in prestigious Jamia Millia Islamia, NIT Uttarakhand, Border Roads etc. He has authored or co-authored over more than 200 papers in various SCI, SCOPUS Indexed and other national, international journals \& conferences. For professional growth, he has travelled more than twenty countries all over the world for paper presentation, session Chair, Workshop etc. He completed a few major projects sponsored by public funding agencies like AICTE, DST etc. He received various awards like Railway Board Medal, Lions Award, UGC Research Associate etc. His research interests include Computer Vision, Image Processing, Artificial Intelligence, Semantic Web, Sensor Networks, Drives, Active Filters, Antennas and Renewable Energy. He is a senior member of IEEE, Fellow IETE, Chartered Engineer IET, and Fellow IE (I).

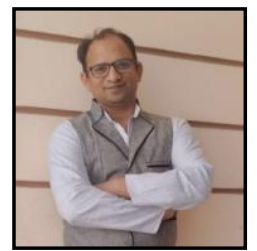

Dr. Anuj Jain (Ph.D, M-Tech, BE), contributed 13 years in teaching and administration activities of various engineering colleges. As a Head of department. His greatest strengths which helped me to achieve a successful head position are my logical and critical thinking. Worked as Associate Professor and Head of Electronics \& Communication. Worked as an Assistant Professor with Electronics \& Communication department at Laxmi Devi Institute of Engineering and Technology, Alwar (Rajasthan). Worked as an Assistant Professor (HOD) and Dean of Engineering with Electrical \& Electronics Engineering Department, at International Institute of Technology \&Buisiness. Sonipat (Haryana). Working as an Acting Professor, SEEE, Lovely Professional University Punjab 